\title{
The Pedestrian Behaviour of Spanish Adolescents
}

Sullman, M. J. M., ${ }^{1}$ Gras, M.E. ${ }^{2}$, Font-Mayolas, S. ${ }^{2}$, Masferrer, L. ${ }^{2}$, Cunill, M. ${ }^{2}$ \& Planes, $\mathrm{M}^{2}$

${ }^{1}$ School of Psychology, University of Hertfordshire, United Kingdom.

${ }^{2}$ Quality of Life Research Institute, University of Girona, Catalonia, Spain

Address for contact:

Dr Mark Sullman

School of Psychology

University of Hertfordshire

College Lane, Hatfield, Hertfordshire, AL10 9AB, UK

Email: M.Sullman@herts.ac.uk

Tel: $\quad+44(0) 1707284625$

Fax $\quad+44(0) 1707285073$ 


\title{
The Pedestrian Behaviour of Spanish Adolescents
}

\begin{abstract}
Adolescent pedestrians are a particularly vulnerable group of road users. This research tested the applicability of the recently developed Adolescent Road user Behaviour Questionnaire (ARBQ) amongst a sample of 2006 Spanish adolescents. Confirmatory Factor Analysis of the full scale found that the original three factors did not adequately fit the data, but an acceptable fit was obtained for the shortened 21-item version of the scale. In line with research from the UK, the present study found that males reported more unsafe road crossing behaviour and playing on the roads, but there was no gender difference for engaging in planned protective behaviour. This research also confirmed that unsafe road crossing behaviour increased with age, while dangerous playing on the road and planned protective behaviours both decreased with age. The present study also confirmed that the ARBQ is a useful tool for investigating the safety-related behaviour of adolescents on the road.
\end{abstract}

Keywords: Adolescents; Behaviour; Road safety; Pedestrians; ARBQ 


\section{Introduction}

In terms of westernised countries Spain has a relatively high number of road deaths. In 2006 there were 4,104 deaths and more than 63,014 injuries on Spanish roads (DGT, 2007). This equates to a fatality rate of $85 / 1,000,000$ people, which is almost twice as high as in the Netherlands $(43 / 1,000,000$ people) and more than three times as high as in Malta $(25 / 1,000,000)$ (European Commission, 2008). However, the risk of being killed or seriously injured in the advent of an accident differs according to the type of road user, with pedestrians being one particularly vulnerable group of road users.

Pedestrian accidents are one of the single biggest causes of injury, disability and death in the developed world. This is particularly the case amongst children, where the problem of pedestrian accidents has been identified as one of the most serious health risks facing children in developed countries (Thomson, Tolmie, Foot, \& Mclaren, 1996). Moreover, injury is the principal cause of child death in all developed nations, accounting for almost $40 \%$ of deaths amongst 1 to 14 year olds, with the single largest proportion of these (41\%) occurring on the road (UNICEF, 2008).

In terms of the Spanish statistics, in 2006 pedestrians were involved in 613 (15\%) deaths and $11,153(18 \%)$ serious injuries, with 5-17 years old accounting for $13 \%$ of all pedestrian injuries and death (DGT, 2007). In order to reduce the number of child pedestrians injured and killed on the roads there is a need to understand the behaviour of adolescents on the roads. 
As would be expected, an extensive amount of research has shown that adolescents engage in risky behaviours on the roads and that these behaviours, along with the failure to engage in personal protective behaviours, increase the risk that they will be killed or injured (e.g. BRAKE, 2004; Elliott \& Baughan, 2004; Sullman \& Mann, 2009; West, Train, Junger, Pickering, Taylor and West, 1998). Therefore, understanding adolescents' on road behaviour is an important first step in the reduction of accidents involving adolescent pedestrians.

Although there is currently no agreed upon framework for investigating the behaviour of adolescent pedestrians the Adolescent Road user Behaviour Questionnaire (ARBQ), developed by Elliott and Baughan (2004), has potential to fill this gap. The original ARBQ contained 43 items and was developed in the UK using information obtained via qualitative descriptions of pedestrian accidents involving children, focus groups with children, and road safety experts. Although the scale focused mainly on pedestrian behaviours, it also included a small number of important behaviours as cyclists and using the road to skateboard and roller blade.

In order to test the ARBQ Elliott and Baughan (2004) surveyed 2,433 English 11-16 year olds and found that the scale was measuring three latent variables. The three factors, which all had good internal reliabilities, were labelled: "unsafe crossing behaviour", "dangerous playing in the road" and "planned protective behaviour". The "unsafe crossing behaviour" factor consisted primarily of behaviours to do with crossing the road in an unsafe manner (e.g. getting partway across the road and having to run the rest of the way to avoid traffic). Dangerous playing on the roads (Factor 2) consisted of behaviours which were mostly to do with playing on the road (e.g. skateboard on the road). In 
contrast to the other two factors, the third factor (planned protective behaviour) was comprised of protective behaviours which were aimed at reducing the risk of being involved in an accident (e.g. using lights when riding a bike). This three way distinction was largely supported in a recently completed study using a sample of New Zealand secondary school students (Sullman \& Mann, 2009).

Although there are substantial differences in the road traffic environment between NZ and the UK, with the most obvious being population density and the ethnic make up of the two populations, there were a large number of similarities in the findings of the two studies. Aside from the substantial agreement in the factor structure, the order of the items (by mean) was also similar, with the position of only two items differing greatly. These two items were: wearing a helmet when riding a bike $\left(3^{\text {rd }}\right.$ in NZ and $27^{\text {th }}$ in the UK) and using a crossing monitor where one was available ( $4^{\text {th }}$ in NZ and $26^{\text {th }}$ in the UK). Although there is an obvious explanation for the difference in the former (using a bike helmet is a legal requirement in NZ, but not the UK), there is no readily available explanation for the latter difference.

Similar relationships were also found in the relationships the ARBQ factors had with the adolescents' demographic variables. In both studies males reported playing on the road significantly more often than females and in neither study were there gender differences in the reported frequency at which adolescents engaged in planned protective behaviour. However, there were also a number of findings which differed between the two studies.

In the UK research, males reported engaging in unsafe road crossing behaviours more often than females (Elliott \& Baughan, 2004), but this was not replicated in the NZ study 
(Sullman \& Mann, 2009). The UK research also reported age differences in the frequency at which the English adolescents reported engaging in the three types of behaviours. They found that 11-12 year olds reported more planned protective behaviours and less unsafe crossing behaviours than both 13-14 year olds and 14-15 year olds. In addition, 13-14 year olds also reported engaging in more dangerous play on the roads than 15-16 year olds. As the NZ research did not include any 11-12 year olds, the only conflicting findings were the absence of a difference between the two groups (13-14 vs. 15-16 year olds) on the dangerous playing on the road factor and the absence of a gender difference for the unsafe road crossing factor.

The frequency at which adolescents engage in the two types of potentially risky behaviours (playing on the road and unsafe road crossing) and planned protective behaviours has important implications for their safety. Information regarding the relationships the three ARBQ factors have with demographic variables, such as age and gender, could help identify who to target with any proposed interventions. This would allow the identification of at risk groups so that interventions could be developed specifically to target them. Finally, this information could also be useful for identifying where additional research resources need to be directed towards.

However, in order for the ARBQ to become an agreed upon framework for investigating the behaviour of adolescents as pedestrians, the scale must be tested in a country and culture more dissimilar than NZ and the UK. The national culture of Spain has been found to differ substantially from those of NZ and the UK on all four (power distance, individualism, masculinity and uncertainty avoidance) of the Hofstede dimensions of national culture that were reported for all three countries (Hofstede, 2008). It would 
therefore be interesting to test whether the three way distinction for adolescent pedestrian behaviour also applies in Spain. Furthermore, it would be interesting to investigate similarities and differences between the three countries. This may help explain why there are large differences in the injury and fatality statistics for the three countries. In addition, if the same behavioural patterns emerge, then perhaps similar interventions may be effective across the three countries.

In summary, this research attempted to describe the road user behaviour of Spanish adolescents. More specifically we investigated the rank ordering of the 43 self-reported behaviours and compared these with previous research from NZ and the UK to investigate similarities and differences between the three countries. Finally, the present study also investigated whether the resultant factors had relationships with the demographic variables measured.

\section{Method}

\section{Participants}

The target population consisted of all secondary students (2180) attending all public schools in the city of Girona (Spain). However, only 2036 students were present during normal class time on the day of the data collection and the data of thirty participants could not be analysed as they had not completed the questionnaire correctly. Therefore, the final sample consisted of 2006 secondary students (92\% participation). The age of the participants ranged from 12 to 17 years old $($ Mean $=13.95, \mathrm{SD}=1.39)$, with $49.8 \%$ being female. 


\section{Materials}

A Spanish version of the Adolescent Road User Behaviour Questionnaire (ARBQ) was used to investigate the behaviour of adolescents on Spanish roads. The ARBQ consists of 43 items which start "As pedestrians, how often do you", followed by examples of road user behaviours. The scale is comprised of three different kinds of behaviours: unsafe crossing behaviour (e.g. "run across a road without looking because you are in a hurry"), playing on the road (e.g. "deliberately run across the road without looking, for a dare") and planned protective behaviour (e.g. "wear bright or protective clothing when out on foot in the dark"). Table 1 shows the full list of these items and the order in which they appeared in the questionnaire. Responses were made on a five point Likert scale $(1=$ Never to $5=$ Very often).

\section{Design and Procedure}

The study was peer reviewed to ensure the study complied with the University's ethical guidelines. Participation was voluntary and adolescents were assured of their anonymity and the confidentiality of their responses. A cross-sectional survey was used to collect the data. After permission had been obtained from the person in charge of each School, the questionnaires were administered to all students present during normal class time. The students answered the ARBQ along with questions about age and gender.

As the original version of the ARBQ was in English, the scale had to be translated into Spanish. Translation of the scale was carried out by two native Spanish speakers and 
these translations were then translated back into English by a native English speaker. No major issues were identified during the translation process.

\section{Results}

\section{Individual items}

Table 1 shows the Spanish means and standard deviations, presented in descending order, for each of the 43 ARBQ items. The table also presents the corresponding data from the New Zealand and English research (Elliott \& Baughan, 2004; Sullman \& Mann, In Press). The behaviour reported most often by the Spanish participants was also the highest in both the English \& NZ samples ("Looking both ways before crossing the road"). This was followed by "Check to make sure traffic has stopped before using a pedestrian crossing", which was also second in both previous studies. The item "Wear a cycle helmet when riding a bike" was the most dissimilarly rated item between the NZ and Spanish adolescents ( $4^{\text {th }}$ in NZ and $26^{\text {th }}$ in Spain). The biggest difference with the UK adolescents was the item "Use a crossing monitor where there is one available", which was rated the $6^{\text {th }}$ most frequently rated behaviour ( $\operatorname{similar}$ to NZ in $7^{\text {th }}$ ) while in the UK it was $26^{\text {th }}$. The behaviour the Spanish adolescents reported more often than the other two samples was "Cross whether traffic is coming or not, thinking the traffic should stop for you $\left(16^{\text {th }}\right.$ in Spain, $28^{\text {th }}$ in NZ and $29^{\text {th }}$ in the UK).

The four least often reported behaviours were almost exactly the same in the three samples. The four least often reported behaviours by Spanish and New Zealand 
adolescents were "Play "chicken" by deliberately running out in front of traffic", "Hold on to a moving vehicle when riding a skateboard or roller blades", "Play chicken by lying down in the road and waiting for cars to come along", and "Deliberately run across the road without looking, for a dare". Three of the bottom four were also in the bottom four of the UK, but the item "Deliberately run across the road without looking, for a dare" was reported more frequently by UK adolescents thank by Spanish adolescents.

\section{[Insert Table 1]}

\section{Factor Analysis}

Confirmatory factor analysis (CFA) was used to investigate whether the factors identified by Elliott and Baughan (2004) were a good fit for the Spanish ARBQ data. LISREL 8.71 was used and the fit was analysed using Maximum Likelihood Estimation procedures. The missing data was treated using the Expectation Maximization (EM) method. The processes began with the three factor model, which included the original 43 items (Elliott and Baughan, 2004). Table 2 presents a summary of the goodness of fit indices. The goodness of fit indices used included the Satorra-Bentler Scaled Chi-Squared statistic and the Non-Normed Fit Index (NNFI), due to the non-normality of the data. The Standardised Root Mean Square (SMRM) and Root Mean-Squared Error of Approximation Measures Residual (RMSEA) were also used.

Although in the first model the NNFI was acceptable (over .95), the SMRM and RMSEA were both higher than desired and so the modification indices were examined. This showed a number of problem pairs of items (see Table 3). Redundant content appeared to be the most likely explanation for these issues, as the pairs of items involved were 
similar. For example, item 11 "Forget to look properly because you are talking to friends who are with you" and item 15 "Forget to look properly because you are thinking about something else" are very similar. Only error correlations between similar items within the same factor were allowed. Allowing these items to be correlated produced a second model which still did not have satisfactory fit indices (see Table 2).

[Insert Table 2]

[Insert Table 3]

The third model tested was the shortened 21-item ARBQ produced by selecting the items that loaded most strongly on the three factors (Elliott and Baughan, 2004). This model also showed an acceptable NNFI, but higher than desired values for the SMRM and RMSEA. When the modification indices were examined they showed problems with seven pairs of items (see Table 4). Allowing these items to be correlated produced a fourth model which had satisfactory fit indices (see Table 2). The correlated version of the 21 item three factor model was therefore selected as the best and is shown in Figure 1.

[Insert Table 4]

Table 5 shows the factor loadings, from the CFA, along with the Construct Reliability Coefficients (Hair, Anderson, Tatham \& Black, 1999) for the fourth model. All factors had good construct reliability (>.70) and the factor loadings were all above .30 .

[Insert Figure 1]

[Insert Table 5] 
Adolescent Road Behaviour by age \& gender

Table 6 shows the mean (and SD) for the three factors, by age and gender. The results of a two way ANOVA (gender $x$ age group) for the three factors found that males reported significantly more unsafe road crossing behaviours $(\mathrm{p}<.001)$ and more dangerous playing in the road $(\mathrm{p}<.001)$. However, there was no difference by gender in the reported engagement in planned protective behaviours.

Table 6 also shows that self-reported unsafe road crossing behaviours increased with age. However, the significant difference was between the 12-13 years old and the two older groups $(\mathrm{p}<.001)$. The difference between the two oldest groups was not significant. Dangerous playing in the road decreased with age, but again the difference was between 12-13 years old group and the two older groups $(\mathrm{p}<.05)$. Planned protective behaviour also decreased with age, with the significant differences again being between the youngest group and the two older groups $(\mathrm{p}<.0001)$. The interaction effects of age and gender on the three different types of ARBQ behaviours were also examined but were not significant.

[Insert Table 6]

[Insert Table 7]

Table 7 shows the means (and SD) for each of the three ARBQ factors, by country, for the total sample and also by gender. It should be noted that the items which comprised this factor in the NZ research (Sullman \& Mann, 2009) were slightly different from those 
that made up the UK and Spanish factors. Therefore the means reported here were recalculated to contain the same items as the present study. One sample t-tests were individually undertaken to test whether the Spanish means were different from the NZ and UK means. This found that the Spanish adolescents reported engaging more often in unsafe road crossing behaviour than the UK adolescents $(\mathrm{p}<.0005)$, but reported the same frequency as NZ adolescents $(\mathrm{p}=.48)$. By gender, the Spanish males reported engaging more often in these behaviours than both the UK $(p<.0005)$ and NZ $(p<.0005)$ males. In addition, Spanish females reported engaging more often in unsafe road crossing behaviour than UK females ( $p<.005)$, but less frequently than the NZ females $(p=.001)$.

In contrast, the Spanish adolescents reported less dangerous playing on the roads than both UK $(\mathrm{p}<.0005)$ and NZ $(\mathrm{p}<.02)$ adolescents. By gender, the Spanish males reported less dangerous playing on the roads than their UK counterparts $(p<.005)$, but more than NZ males $(p=.003)$. Spanish females reported less playing on the roads than both UK $(\mathrm{p}<.0005)$ and $\mathrm{NZ}$ females $(\mathrm{p}<.0005)$.

The planned protective behaviours were clearly more frequently reported by NZ adolescents, than Spanish adolescents, for the whole sample $(\mathrm{p}<.0005)$ and for both genders $(\mathrm{p}<.0005)$. Compared to the UK, Spanish adolescents also reported engaging in planned protective behaviours less often $(\mathrm{p}<.01)$, but this difference only remained significant for females $(p=.005)$, but not males $(p=.53)$.

\section{Discussion}

The present study investigated the road user behaviour of Spanish adolescents and made a substantial number of findings which were in agreement with previous research. Firstly, 
there were considerable similarities in the ordering of the 43 behaviours in the Spanish sample to those found in both NZ and the UK. The two most frequently reported behaviours ("Looking both ways before crossing the road" \& "Check to make sure traffic has stopped before using a pedestrian crossing") were also the two most frequently reported in both the UK and NZ samples. There was also a high degree of similarity in the behaviours which were least frequently reported, with the lowest five in Spain and NZ being exactly the same and four of the five also being the lowest in the UK sample. There were, however, a number of differences between the three countries, with the Spanish adolescents reporting a much lower level of cycle helmet use than the NZ adolescents (using a cycle helmet is a legal requirement in NZ). In contrast, the Spanish adolescents engaged more often, than the English and NZ adolescents, in the behaviour "Cross whether traffic is coming or not, thinking the traffic should stop for you". This is an interesting finding and possibly points to evidence of a cultural difference with the other two English speaking countries, where this behaviour is less common.

Although the bottom five behaviours were reported infrequently, in total $15 \%$ of Spanish adolescents reported that they "Play "chicken" by deliberately running out in front of traffic" at some stage. Very similar proportions were also found for the other four least frequently reported behaviours. These findings are particularly disturbing, as safety experts rated these five behaviours as being extremely risky (Elliott \& Baughan, 2003). The challenge for road safety experts, therefore, is to continue to reduce the number of adolescents (and other road users) engaging in these five very dangerous behaviours, along with other potentially risky behaviours, while at the same time promoting selfprotective behaviours. 
The present research also found that confirmatory factor analysis of the Spanish ARBQ broadly supported the original three factor solution found in the UK sample (Elliott \& Baughan, 2004). However, unfortunately it was not possible to produce a satisfactory fit for the three factor model with 43 items. In contrast, satisfactory goodness of fit indices were obtained for the short 21 -item scale. This finding is very important, as the much shorter 21-item scale is a more practical research tool which can be used more easily in combination with other research instruments.

The most obvious feature of the international comparison was the fact that the NZ adolescents (both male and female) reported substantially higher engagement in planned protective behaviours than the Spanish and UK adolescents. This seems to be mainly due to the high level that $\mathrm{NZ}$ adolescents reported using a cycle helmet, as none of the other items which comprised this factor differed substantively. Another notable feature is the fact that Spanish males appear to engage in more negative behaviours (unsafe crossing \& dangerous playing on the road) and less positive behaviours (unplanned protective behaviours) than NZ males. The Spanish females also engaged in planned protective behaviours less often than the UK and NZ females. Perhaps this is related to lower risk perceptions or higher levels of some other individual differences (e.g. sensation seeking) which are related to risky behaviours and engagement in protective behaviours. Regardless of the cause, this finding should be concerning for Spanish safety authorities, as the rate at which Spanish 1-14 year olds are killed on the roads $(4.0 / 100,000)$ is considerably higher than in the UK $(2.9 / 100,000)$ (UNICEF, 2008). Therefore, the challenge for Spain should be to equal, or better, the fatality statistics of the UK. 
The relationships the ARBQ factors had with age and gender also showed a high degree of similarity with previous research, particularly that from the UK. The pattern of findings by gender directly replicated Elliott \& Baughan’s (2004) findings, in that males reported significantly higher levels of unsafe crossing behaviour and dangerous playing on the road, but there was no difference between males and females on the planned protective behaviour factor. These findings also largely agree with the NZ research, in there being no gender difference on the planned protective behaviour factor and that males reported more dangerous playing on the roads (Sullman \& Mann, 2009).

The finding that males engage in more risky on road behaviours in Spain, England and to some extent NZ is also in agreement with previous research using different instruments (e.g. BRAKE, 2004; Byrnes, Miller \& Schafer, 1999; Granié, 2007; Morrongiello \& Dawber, 1999; Waylen \& McKenna, 2008; West et al., 1998). Moreover, these findings are also supported by the accident statistics, which have shown that most of the adolescents killed and injured on the roads are male (e.g. DFT, 2008; DGT, 2007; Poudel-Tandukar Nakahara, Ichikawa, Poudel, \& Wakai., 2006; Sullman \& Mann, 2009; Twisk, 2007).

The relationships the three ARBQ factors had with age were also very similar to those previously found amongst English adolescents (Elliott \& Baughan, 2004). In agreement with the English research this study found age differences on all three factors, with unsafe road crossing increasing with age, while playing on the roads and protective behaviours both decreased with age. These findings may appear to be counter intuitive, as in most areas of road safety, risky behaviours decrease with age. However, the increase in unsafe road crossing behaviour and reduced engagement in personal protective 
behaviours may be partially explained by the fact that at this time in a child's life their exposure to risk increases, while the amount of adult supervision decreases (Lynam \& Harland, 1992). In contrast to the other two factors, dangerous playing on the road decreased with age. Therefore, the present research seems to highlight the need for safety interventions to target males, in general, and the two older age groups in order to increase their personal protective behaviours and to reduce their engagement in unsafe road crossing behaviour. Furthermore, the road safety countermeasures need to target dangerous playing on the road amongst the 12-13 year olds.

Although it may appear reasonable to argue that safety improvements would eventuate by making positive changes in the three types of behaviours measured by the ARBQ, future research is needed to investigate whether the three ARBQ factors are related to important safety outcome measures, such accidents and near misses. This would be an obvious way to clearly demonstrate that making positive changes in the behaviours measured by the ARBQ would lower the adolescents' risk of being killed or injured on the road. Furthermore, although understanding the relationships the ARBQ factors have with demographic variables is potentially useful for targeting road safety interventions, it does not identify the causes of these behaviours. Therefore, future research is needed into the psychological precursors of the three types of ARBQ behaviours. Thrill seeking behaviour, unrealistic optimism and mild social deviance have all been shown to be associated with risky behaviours in other domains (e.g. Scott-Parker, Watson, \& King, 2009; Sullman, Meadows, \& Pajo, 2002; Waylen \& McKenna, 2008; West et al., 1998) and may prove to be useful starting point. A more clear understanding of the psychological mechanisms underlying these behaviours may assist in the development of methods to influence adolescents' on road behaviour. 
The study reported here clearly had a number of methodological limitations. The most obvious is the possibility of sampling bias. As all participants attended secondary schools in the city of Girona, it is possible that they differ significantly in some way from the general population of Spain. However, confidence in this data can be drawn from the fact that a large number of the results were similar to those found in the NZ and UK research, both of which used less regionally focused samples.

In summary, this research has confirmed the applicability of the ARBQ to Spain, a country and culture which is more distinctive than the UK and NZ. The present study supported the factor structure of the 21-item scale, and found that Spanish males were more likely than females to engage in unsafe road crossing behaviour and dangerous playing on the road. This research also found that younger Spanish adolescents engaged more often in dangerous playing on the roads, while older adolescents were more likely to put themselves at risk by failure to elicit personal protective behaviours, or by engaging in unsafe road crossing behaviours. Overall, the present study concludes that the ARBQ appears to be a useful framework for investigating the behaviour of adolescents on the road.

\section{Acknowledgements}

The authors would like to thank the schools and pupils that participated in the study. 


\section{References}

BRAKE (2004). Hard facts - statistics and research: Survey of 11-14 year olds. (http://www.brake.org.uk/)

Byrnes, J., Miller, D., Schafer, W. (1999). Gender differences in risk taking: A metaanalysis. Psychological Bulletin, 125, 367-383.

DFT (2008). Child Fact Sheet - Child Casualties in Road Accidents Great Britain: 2006 $\begin{array}{llllllll}\text { Retrieved from the World Wide Web July } 7 & 2008 \text { from }\end{array}$ http://www.dft.gov.uk/162259/162469/221412/221549/227755/328843/childfact20 06.pdf

DGT (2007). Anuario estadístico de accidentes 2006. Retrieved from the World Wide Web http://www.dgt.es/was6/portal/contenidos/documentos/seguridad_vial/estadistica/ac cidentes_30dias/anuario_estadistico/anuario_estadistico001.pdf

Elliott, M.A., \& Baughan, C.J. (2004). Developing a self-report method for investigating adolescent road user behaviour. Transportation Research Part F, 7, 373-393.

European Commission (2008). Road Safety Scoreboard. Retrieved July 72008 from the World Wide Web http://ec.europa.eu/transport/roadsafety_library/scoreboard/scoreboard.pdf

Granié, M-A. (2007). Gender differences in preschool children's declared and behavioural compliance with pedestrian rules. Transportation Research Part F, 10, $371-382$.

Hair, J.F., Anderson, R.E., Tatham, R.L. \& Black, W.C. (1999) Análisis Multivariante. Prentice-Hall: Madrid. 
Hofstede, G. (2008). Geert Hofstede Cultural Dimensions. Retrieved July 102008 from the World Wide Web http://www.geerthofstede.com/hofstede_dimensions.php?culture $1=83 \&$ culture $2=63$

Lynam, D., \& Harland, D. (1992). Child pedestrian safety in the UK. Berlin: VTI/FERSI Conference.

Morrongiello, B., \& Dawber, T. (1999). Parental influences on toddlers' injury-risk behaviors: Are sons and daughters socialised differently? Journal of Applied Developmental Psychology, 27, 227-251.

Poudel-Tandukar, K., Nakahara, S., Ichikawa, M., Poudel, K.C., \& Wakai, S. (2006). Relationship between mechanisms and activities at the time of pedestrian injury and activity limitation among school adolescents in Kathmandu, Nepal. Accident Analysis \& Prevention, 38, 1058-1063.

Scott-Parker, B., Watson, B., \& King, M. J. (2009). Understanding the psychosocial factors influencing the risky behaviour of young drivers. Transportation Research Part F, 12, 470-482.

Sullman, M.J.M., \& Mann, H. (2009). Adolescent Road User Behaviour amongst New Zealand Adolescents. Transportation Research Part F, 12, 494-502.

Sullman, M.J.M., Meadows, M.L., \& Pajo, K. (2002). Aberrant driving behaviours amongst New Zealand truck drivers. Transportation Research Part F, 5, 217-232.

Twisk, D. (2007). Jóvenes conductors: el camino hacia la seguridad. In: Real Automóvil Club de Cataluña (ed.). Jóvenes y conducción: un derecho y una responsabilidad. Barcelona: RACC.

Thomson, J. A., Tolmie, A., Foot, H. C., \& Mclaren, B. (1996). Child development and the aims of Road Safety Education: A Review and Analysis. Road Safety Research Report No.1, Department of Transport, UK. 
UNICEF. (2001). A League Table of Child Deaths by Injury in Rich Nations. Retrieved July 102008 from the World Wide Web http://www.unicef-icdc.org

Waylen, A.E., \& McKenna, F.P. (2008). Risky attitudes towards road use in pre-drivers. Accident Analysis \& Prevention, 40, 905-911.

West, R., Train, H., Junger, M., Pickering, A., Taylor, E., \& West, A. (1998). Childhood accidents and their relationship with problem behaviour. DETR Report No 7. London: The Stationery Office. 
Table 1

ARBQ behaviour items means and standard deviations for Spain, NZ \& English

\begin{tabular}{|c|c|c|c|c|c|c|c|}
\hline \multirow[t]{2}{*}{ No. } & \multirow[t]{2}{*}{ Item (How often do you...) } & \multicolumn{2}{|c|}{ Spain } & \multicolumn{2}{|c|}{ NZ } & \multicolumn{2}{|c|}{ England } \\
\hline & & $M$ & SD & $M$ & SD & $M$ & SD \\
\hline 1 & Look both ways before crossing the road & 4.07 & 1.08 & 4.17 & .95 & 4.08 & 1.07 \\
\hline 2 & $\begin{array}{l}\text { Check to make sure traffic has stopped before using a pedestrian } \\
\text { crossing }\end{array}$ & 3.67 & 1.15 & 3.73 & 1.15 & 3.46 & 1.30 \\
\hline 3 & Cross at a place that is well lit when it is dark & 3.48 & 1.18 & 3.13 & 1.08 & 3.32 & 1.16 \\
\hline 4 & Keep looking and listening until you get all the way across the road & 3.34 & 1.24 & 3.24 & 1.17 & 3.26 & 1.26 \\
\hline 11 & $\begin{array}{l}\text { Forget to look properly because you are talking to friends who are } \\
\text { with you }\end{array}$ & 3.05 & 1.12 & 2.80 & 1.06 & 2.65 & 1.15 \\
\hline 10 & $\begin{array}{l}\text { Cross between parked cars when there is a safer place to cross } \\
\text { nearby }\end{array}$ & 2.92 & 1.07 & 2.88 & 1.03 & 2.66 & 1.16 \\
\hline 26 & Use a crossing monitor where there is one available & 2.92 & 1.35 & 3.27 & 1.27 & 2.06 & 1.20 \\
\hline 6 & $\begin{array}{l}\text { Get part way across the road and then have to run the rest of the } \\
\text { way to avoid traffic }\end{array}$ & 2.88 & 1.04 & 3.01 & 1.04 & 2.82 & 1.14 \\
\hline 8 & Not bother walking to a nearby crossing to cross the road & 2.85 & 1.14 & 3.10 & 1.13 & 2.72 & 1.22 \\
\hline 12 & Walk in single file on roads without pavements & 2.81 & 1.41 & 2.40 & 1.16 & 2.54 & 1.38 \\
\hline 17 & Cross from behind a stationary vehicle & 2.79 & 1.12 & 2.83 & 1.05 & 2.35 & 1.17 \\
\hline 7 & Cross without waiting for the "green man" & 2.76 & 1.58 & 2.42 & 1.23 & 2.76 & 1.25 \\
\hline 9 & See a small gap in traffic and "go for it" * & 2.73 & 1.13 & 3.14 & 1.08 & 2.69 & 1.30 \\
\hline
\end{tabular}




\begin{tabular}{|c|c|c|c|c|c|c|c|}
\hline \multirow[t]{2}{*}{ No. } & \multirow[t]{2}{*}{ Item (How often do you...) } & \multicolumn{2}{|c|}{ Spain } & \multicolumn{2}{|c|}{ NZ } & \multicolumn{2}{|c|}{ England } \\
\hline & & $M$ & SD & $M$ & SD & $M$ & SD \\
\hline 16 & Make traffic slow down or stop to let you cross & 2.66 & 1.14 & 2.22 & 1.05 & 2.43 & 1.24 \\
\hline 21 & Not look because you cannot hear any traffic around & 2.59 & 1.23 & 2.65 & 1.16 & 2.25 & 1.22 \\
\hline 29 & $\begin{array}{l}\text { Cross whether traffic is coming or not, thinking the traffic should } \\
\text { stop for you }\end{array}$ & 2.55 & 1.23 & 2.15 & 1.12 & 1.99 & 1.21 \\
\hline 13 & Walk facing the traffic when on roads without pavements & 2.51 & 1.23 & 2.89 & 1.19 & 2.51 & 1.36 \\
\hline 15 & $\begin{array}{l}\text { Forget to look properly because you are thinking about something } \\
\text { else }\end{array}$ & 2.46 & 1.01 & 2.47 & 0.99 & 2.44 & 1.08 \\
\hline 5 & Use lights on your bike when it is dark & 2.37 & 1.56 & 2.64 & 1.53 & 2.84 & 1.62 \\
\hline 24 & $\begin{array}{l}\text { Not notice a car pulling out (say from a driveway) and walk in front } \\
\text { of it? }\end{array}$ & 2.36 & 1.09 & 2.30 & 1.03 & 2.20 & 1.03 \\
\hline 23 & Walk in the road rather than on the pavement? & 2.29 & 0.96 & 2.41 & 1.00 & 2.22 & 1.07 \\
\hline 18 & Have to stop quickly or turn back to avoid traffic & 2.27 & 1.01 & 2.23 & 0.94 & 2.34 & 1.07 \\
\hline 14 & $\begin{array}{l}\text { Cross when you cannot see both ways very well (like on a bend or } \\
\text { top of hill) }\end{array}$ & 2.25 & 0.96 & 2.45 & 0.94 & 2.49 & 1.10 \\
\hline 19 & $\begin{array}{l}\text { Think it is OK to cross safely, but a car is coming faster than you } \\
\text { thought }\end{array}$ & 2.17 & 0.98 & 2.38 & 0.92 & 2.34 & 1.12 \\
\hline 25 & Run across a road without looking because you are in a hurry & 2.17 & 1.07 & 2.10 & 1.04 & 2.20 & 1.22 \\
\hline 27 & Wear a cycle helmet when riding a bike & 2.03 & 1.41 & 3.70 & 1.40 & 2.03 & 1.40 \\
\hline 28 & Use a mobile phone and forget to look properly & 2.01 & 1.04 & 2.07 & 1.15 & 2.03 & 1.15 \\
\hline 30 & Climb over barriers or railings that separate the road from the & 1.95 & 1.08 & 1.94 & 1.09 & 1.97 & 1.21 \\
\hline
\end{tabular}




\begin{tabular}{|c|c|c|c|c|c|c|c|}
\hline \multirow[t]{2}{*}{ No. } & \multirow[t]{2}{*}{ Item (How often do you...) } & \multicolumn{2}{|c|}{ Spain } & \multicolumn{2}{|c|}{ NZ } & \multicolumn{2}{|c|}{ England } \\
\hline & & $M$ & SD & $M$ & SD & $M$ & SD \\
\hline & pavement & & & & & & \\
\hline 20 & Hang around in the road talking to friends & 1.90 & 1.00 & 2.43 & 1.15 & 2.27 & 1.23 \\
\hline 22 & Run around on the road (e.g. when playing cricket or football) & 1.80 & 1.04 & 2.21 & 1.14 & 2.24 & 1.29 \\
\hline 35 & Wear bright or reflective clothing when riding a bike in the dark* & 1.78 & 1.23 & 1.95 & 1.26 & 1.79 & 1.24 \\
\hline 31 & Not notice an approaching car when playing games in the road & 1.76 & 0.93 & 2.21 & 1.24 & 1.93 & 1.11 \\
\hline 32 & Run into the road to get a ball, without checking for traffic & 1.76 & 0.95 & 1.83 & 0.96 & 1.87 & 1.09 \\
\hline 34 & Ride on a skateboard (or roller-skates/roller-blades) in the road* & 1.72 & 1.04 & 1.68 & 1.05 & 1.85 & 1.26 \\
\hline 36 & Wear reflective clothing when out on foot in the dark* & 1.58 & 1.00 & 1.69 & 1.04 & 1.67 & 1.07 \\
\hline 39 & Wear reflective clothing when crossing a road* & 1.41 & 0.80 & 1.60 & 0.91 & 1.49 & 0.93 \\
\hline 33 & Cross less than an hour after drinking alcohol* & 1.46 & 0.91 & 1.58 & 0.97 & 1.87 & 1.28 \\
\hline 41 & Hold on to a moving vehicle when riding a bike* & 1.42 & 0.92 & 1.36 & .82 & 1.36 & .89 \\
\hline 38 & $\begin{array}{l}\text { Ride out into the road on a skateboard without thinking to check for } \\
\text { traffic* }\end{array}$ & 1.31 & 0.71 & 1.36 & .73 & 1.50 & .95 \\
\hline 43 & $\begin{array}{l}\text { Play "chicken" by lying down in the road and waiting for cars to } \\
\text { come along* }\end{array}$ & 1.27 & 0.74 & 1.35 & .87 & 1.35 & .89 \\
\hline 37 & Deliberately run across the road without looking, for a dare* & 1.24 & 0.66 & 1.34 & .81 & 1.51 & .95 \\
\hline 40 & $\begin{array}{l}\text { Hold on to a moving vehicle when riding a skateboard/roller- } \\
\text { skates/roller-blades* }\end{array}$ & 1.24 & 0.68 & 1.35 & .81 & 1.38 & .91 \\
\hline 42 & Play "chicken" by deliberately running out in front of traffic* & 1.23 & 0.68 & 1.33 & .77 & 1.36 & .88 \\
\hline
\end{tabular}

Note: Scale ranges from $1=$ Never to $5=$ Very Often; $*$ reversed for all subsequent analyses. 
Table 2

Summary of goodness of fit indices for the CFA models

\begin{tabular}{|c|c|c|c|c|c|}
\hline Model & $\mathrm{X}^{2}$ (Satorra-Bentler) & NNFI & SMRM & RMSEA & C.I. $90 \%$ \\
\hline \multirow[t]{2}{*}{ First model fitted* } & 4247.2 & .96 & .11 & .060 & $.058: .061$ \\
\hline & $v=857$ & & & $\mathrm{p}<.01$ & \\
\hline \multirow[t]{2}{*}{ Second model fitted ${ }^{* *}$} & 1846.3 & .97 & .09 & .056 & $.046: .066$ \\
\hline & $v=846$ & & & $\mathrm{p}<.01$ & \\
\hline \multirow[t]{2}{*}{ Third model fitted $* * *$} & 1764.2 & .96 & .09 & .064 & $.061: .067$ \\
\hline & $v=186$ & & & $\mathrm{p}<.01$ & \\
\hline \multirow[t]{2}{*}{ Fourth model fitted $* * * *$} & 661.3 & .98 & .08 & .037 & $.034: .040$ \\
\hline & $v=179$ & & & $\mathrm{p}=1$ & \\
\hline
\end{tabular}

* 43 items with all the errors uncorrelated

** 43 items with 11 error covariances added.

*** 21 items with all the errors uncorrelated

$* * * * 21$ items with 7 error covariances added. 
Table 3

Error correlations between pairs with similar contents within the same factor for the second model.

\begin{tabular}{ll}
\hline Pairs of items & Error correlation \\
\hline 11 and 15 & 0.062 \\
11 and 28 & 0.072 \\
15 and 28 & 0.046 \\
34 and 38 & 0.097 \\
34 and 40 & 0.159 \\
34 and 42 & 0.051 \\
38 and 40 & 0.093 \\
38 and 42 & 0.045 \\
38 and 43 & 0.022 \\
40 and 41 & 0.187 \\
41 and 42 & 0.125 \\
\hline
\end{tabular}


Table 4

Error correlations between pairs with similar contents within the same factor for the fourth model.

\begin{tabular}{ll}
\hline Pairs of items & Error correlation \\
\hline 5 and 27 & 0.188 \\
11 and 15 & 0.030 \\
32 and 38 & 0.037 \\
34 and 38 & 0.064 \\
34 and 40 & 0.089 \\
38 and 40 & 0.081 \\
40 and 41 & 0.125 \\
\hline
\end{tabular}




\section{Table 5}

Factor loadings from the confirmatory factorial analysis
Item No.
Item
Factor loading

Factor 1. Unsafe road crossing behaviour construct reliability coefficient $=.74$

25 Run across a road without looking

.71

because you are in a hurry.

$9 \quad$ See a small gap in traffic and "go for it."

$6 \quad$ Get party way across the road and have to

run the rest of the way to avoid traffic.

19 Think it is OK to cross safely, but a car is

coming faster than you thought.

15 Forget to look properly because you are

thinking about something else.

11 Forget to look properly because you are

talking to friends who are with you.

10 Cross from between parked cars when

there is a safer place to cross nearby.

29 Cross whether traffic is coming or not,

thinking the traffic should stop for

you.

Factor 2. Dangerous playing crossing behaviour construct reliability coefficient $=.85$

42 Play "chicken" by deliberately running

.92

out in front of traffic.

$43 \quad$ Play "chicken" by lying down in the road

.86

and waiting for cars to come along. 
37 Deliberately run across the road without

looking, for a dare.

$40 \quad$ Hold on to a moving vehicle when riding

a skateboard/ roller-skates/roller-blades.

$41 \quad$ Hold on to a moving vehicle when riding

.68

a bike.

38 Ride out into the road on a skateboard

without thinking to check for traffic.

32 Run into the road to get a ball, without

checking for traffic.

34 Ride on skateboard (or roller-

skates/roller-blades) in the road.

Factor 3. Planned protective behaviour construct reliability coefficient $=.70$

36 Wear bright or reflective clothing when out on foot in the dark.

39 Wear reflective clothing when near the .80 road.

35 Wear bright or reflective clothing when .66 riding a bike in the dark.

$27 \quad$ Wear a cycle helmet when riding a bike.

$5 \quad$ Use lights on your bike when it is dark .34 
Table 6

Means of the three types of road user behaviours, by age and gender

\begin{tabular}{clccc}
\hline Factor & & $12-13$ & $14-15$ & $16-17$ \\
& & Mean (SD) & Mean (SD) & Mean (SD) \\
\hline Unsafe road crossing behaviour & Male & $2.56(.64)$ & $2.72(.65)$ & $2.73(.70)$ \\
& Female & $2.47(.60)$ & $2.60(.66)$ & $2.70(.56)$ \\
Dangerous playing in the road & Male & $1.60(.67)$ & $1.56(.65)$ & $1.47(.62)$ \\
& Female & $1.32(.36)$ & $1.25(.42)$ & $1.22(.40)$ \\
Planned protective behaviour & Male & $2.01(.85)$ & $1.76(.78)$ & $1.68(.80)$ \\
& Female & $2.04(.89)$ & $1.79(.80)$ & $1.59(.60)$ \\
\hline
\end{tabular}


Table 7

International comparison by gender

\begin{tabular}{lcccccccccc}
\hline \multicolumn{1}{c}{ Factor } & \multicolumn{3}{c}{ Males } & \multicolumn{3}{c}{ Females } & \multicolumn{3}{c}{$\begin{array}{c}\text { Total } \\
\text { sample }\end{array}$} \\
& & & & & & & & \\
& Spain & NZ & UK & Spain & NZ & UK & Spain & NZ & UK \\
\hline Unsafe road & 2.67 & 2.57 & 2.53 & 2.56 & 2.63 & 2.41 & 2.61 & 2.60 & 2.47 \\
crossing & $(.65)$ & $(.60)$ & $(.83)$ & $(.63)$ & $(.59)$ & $(.75)$ & $(.64)$ & $(.60)$ & $(.79)$ \\
behaviour & & & & & & & & & \\
Dangerous & 1.57 & 1.50 & 1.66 & 1.27 & 1.41 & 1.35 & 1.42 & 1.45 & 1.51 \\
playing in the & $(.66)$ & $(.65)$ & $(.78)$ & $(.39)$ & $(.60)$ & $(.54)$ & $(.56)$ & $(.63)$ & $(.69)$ \\
road & & & & & & & & & \\
Planned & 1.85 & 2.33 & 1.87 & 1.86 & 2.30 & 1.93 & 1.85 & 2.31 & 1.90 \\
protective & $(.82)$ & $(.84)$ & $(.83)$ & $(.82)$ & $(.90)$ & $(.86)$ & $(.82)$ & $(.87)$ & $(.85)$ \\
behaviour & & & & & & & & & \\
\hline
\end{tabular}

Note: NZ means were recalculated using the same items as the Spanish factors 
Figure 1

Confirmatory factor analysis of the 21-item ARBQ

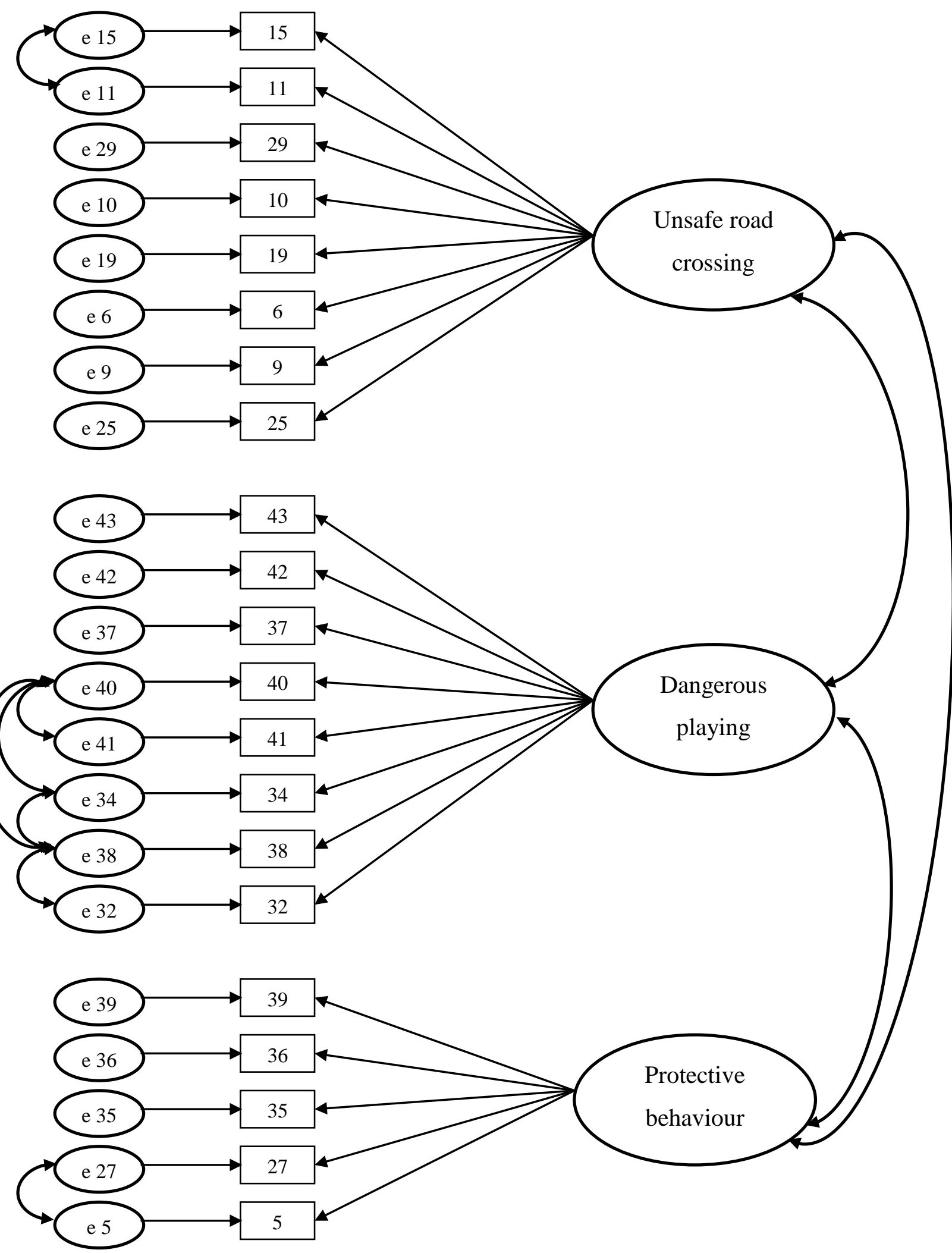

